\title{
Implementation strategies to improve statin utilization in individuals with hypercholesterolemia: a systematic review and meta-analysis
}

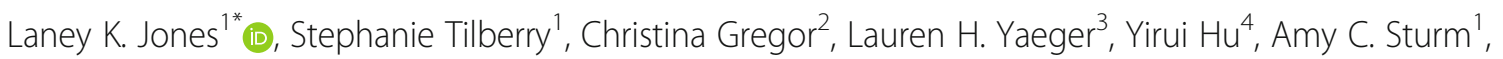

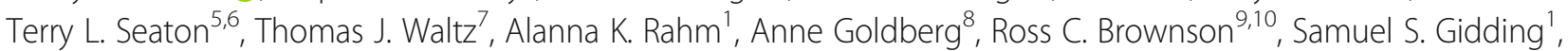
Marc S. Williams ${ }^{1}$ and Michael R. Gionfriddo ${ }^{2}$

\begin{abstract}
Background: Numerous implementation strategies to improve utilization of statins in patients with hypercholesterolemia have been utilized, with varying degrees of success. The aim of this systematic review is to determine the state of evidence of implementation strategies on the uptake of statins.

Methods and results: This systematic review identified and categorized implementation strategies, according to the Expert Recommendations for Implementing Change (ERIC) compilation, used in studies to improve statin use. We searched Ovid MEDLINE, Embase, Scopus, Cochrane Database of Systematic Reviews, Cochrane Central Register of Controlled Trials, and Clinicaltrials.gov from inception to October 2018. All included studies were reported in English and had at least one strategy to promote statin uptake that could be categorized using the ERIC compilation. Data extraction was completed independently, in duplicate, and disagreements were resolved by consensus. We extracted LDL-C (concentration and target achievement), statin prescribing, and statin adherence (percentage and target achievement). A total of 258 strategies were used across 86 trials. The median number of strategies used was 3 (SD 2.2, range 1-13). Implementation strategy descriptions often did not include key defining characteristics: temporality was reported in 59\%, dose in 52\%, affected outcome in 9\%, and justification in 6\%. Thirty-one trials reported at least 1 of the 3 outcomes of interest: significantly reduced LDL-C (standardized mean difference $[\mathrm{SMD}]-0.17,95 \% \mathrm{Cl}-0.27$ to $-0.07, p=0.0006$; odds ratio [OR] $1.33,95 \% \mathrm{Cl} 1.13$ to $1.58, p=0.0008$ ), increased rates of statin prescribing (OR $2.21,95 \% \mathrm{Cl} 1.60$ to $3.06, p<0.0001$ ), and improved statin adherence (SMD $0.13,95 \% \mathrm{Cl} 0.06$ to $0.19 ; p=0.0002 ; \mathrm{OR} 1.30,95 \% \mathrm{Cl} 1.04$ to $1.63, p=0.023)$. The number of implementation strategies used per study positively influenced the efficacy outcomes.
\end{abstract}

Conclusion: Although studies demonstrated improved statin prescribing, statin adherence, and reduced LDL-C, no single strategy or group of strategies consistently improved outcomes.

Trial registration: PROSPERO CRD42018114952.

Keywords: Statin, Hypercholesterolemia, Implementation strategies, Uptake, Meta-analysis

\footnotetext{
*Correspondence: Ljones14@geisinger.edu

'Genomic Medicine Institute, Geisinger, 100 N Academy Ave., Danville, PA

17822, USA

Full list of author information is available at the end of the article
}

C C The Author(s). 2021 Open Access This article is licensed under a Creative Commons Attribution 4.0 International License, which permits use, sharing, adaptation, distribution and reproduction in any medium or format, as long as you give appropriate credit to the original author(s) and the source, provide a link to the Creative Commons licence, and indicate if changes were made. The images or other third party material in this article are included in the article's Creative Commons licence, unless indicated otherwise in a credit line to the material. If material is not included in the article's Creative Commons licence and your intended use is not permitted by statutory regulation or exceeds the permitted use, you will need to obtain permission directly from the copyright holder. To view a copy of this licence, visit http://creativecommons.org/licenses/by/4.0/. The Creative Commons Public Domain Dedication waiver (http://creativecommons.org/publicdomain/zero/1.0/) applies to the data made available in this article, unless otherwise stated in a credit line to the data. 


\section{Contributions to the literature}

- A variety of implementation strategies have been used to promote statin uptake.

- Lack of generalizability of implementation strategies to improve statin use is due in part to lack of detailed reporting of these strategies in the literature.

- No single implementation strategy appears to be associated with improved outcomes when compared with others.

- Multiple implementation strategies are likely to be required to improve statin utilization.

\section{Introduction}

Statin medications reduce low-density lipoprotein cholesterol (LDL-C) blood concentrations and cardiovascular events in patients with hypercholesterolemia, and guidelines recommend statin therapy to lower LDL-C in patients who are at risk for developing or have known atherosclerotic cardiovascular disease [1]. Despite evidence for the benefits of statins, the medications are widely underutilized [2-6]. Previous studies highlight both patient- and prescriber-barriers to statin use including side effects, competing medical conditions, busy clinics, and patient reluctance affecting adherence to prescribed medications [7-9]. Lack of adherence is associated with increased mortality in a dose dependent relationship [10].

Implementation strategies can be used to promote the uptake of interventions, such as statin therapy, and are defined as "methods or techniques used to enhance the adoption, implementation, and sustainability of a clinical program or practice" [11]. Numerous implementation strategies have been attempted to improve utilization of statins, all with varying degrees of success. These studies have targeted a variety of actors (e.g., patients, clinicians, or systems) and employed a variety of implementation strategies (e.g., education, reminders, or financial incentives). A computer-based clinical decision support system to aid in prescribing of evidence-based treatment for hyperlipidemia, which targeted clinicians, was found to significantly reduce blood LDL-C concentrations [12]. However, when providing financial incentives to providers, patients, or both, a study found that only the combination incentive was successful in reducing LDL-C levels to target [13]. The absolute and comparative effectiveness of these strategies, however, is unclear. Knowing which strategies are most effective can facilitate the uptake of statins and lead to reduce mortality.

To address this issue, we aimed to address the following key questions:
1. What implementation strategies have been used to promote the uptake of statins?

2. How completely are the implementation strategies utilized reported in studies designed to promote statin uptake?

3. Which implementation strategy, or combination of strategies, is (are) the most effective at promoting the uptake of statins?

We conducted a systematic review of studies aimed at improving statin use and categorized implementation strategies by the Expert Recommendations for Implementing Change (ERIC) compilation [14]. Our primary objective was to better understand the impact of specific implementation strategies on the utilization of statins in patients with hypercholesterolemia. Our secondary objective was to evaluate statin adherence, statin prescribing, and lowering of LDL-C after intervention.

\section{Methods}

This registered (PROSPERO CRD42018114952) systematic review adhered to the reporting guidelines of the Preferred Reporting Items for Systematic Reviews and Meta-analyses (PRISMA) statement [15].

\section{Search strategy}

A medical librarian (L.H.Y.) searched the literature for records including the concepts of hypercholesterolemia, hyperlipidemia, and statins. The search strategies used a combination of keywords and controlled vocabulary and searched the following databases from inception to October 2018: MEDLINE, Embase, Scopus, Cochrane Database of Systematic Reviews, Cochrane Central Register of Controlled Trials, and Clinicaltrials.gov. References were imported into Endnote ${ }^{\mathrm{Tm}}$ and duplicates were identified and removed. An example of the search string can be found in Table 1 and the fully reproducible search strategies for each database can be found in Additional file 1: Appendix 1.

\section{Study selection}

We included studies reported in English, regardless of the country where the study was conducted, that had at least one strategy promoting statin uptake that could be categorized using the ERIC compilation $[14,16]$. Seven manuscripts were excluded for this reason. The ERIC compilation was created so that researchers have a standardized way to name, define, and categorize implementation strategies. The ERIC compilation was selected for use in this review because the implementations strategies in the included articles most closely matched the ERIC taxonomy compared to other available choices [17]. For key questions 1 and 2, we did not limit inclusion based on study design or outcome. For key question 3, we 
Table 1 Example search string

\begin{tabular}{ll}
\hline Database & Search string \\
\hline Embase & ('hypercholesterolemia'/exp OR 'familial hypercholesterolemia'/exp OR hypercholesterolemia:ti,ab,kw OR cholesteremia:ti,ab,kw OR \\
& cholesterinemia:ti,ab,kw OR cholesterolemia:ti,ab,kw OR hypercholesteremia:ti,ab,kw OR hypercholesterinaemia:ti,ab,kw OR \\
& hypercholesterinemia:ti,ab,kw OR hypercholesterolaemia:ti,ab,kw OR ('high cholesterol' NEAR/1 level*):ti,ab,kw) OR ((elevated NEAR/1 \\
& cholesterol*):ti,ab,kw) OR 'hyperlipidemia'/exp OR 'familial hyperlipemia'/exp OR hyperlipemia*:ti,ab,kw OR hyperlipaemia:ti,ab,kw OR \\
& hyperlipemia:ti,ab,kw OR hyperlipidaemia:ti,ab,kw OR hyperlipidaemias:ti,ab,kw OR hyperlipidemias:ti,ab,kw OR hyperlipidemic:ti,ab,kw OR \\
& lipaemia:ti,ab,kw OR lipemia:ti,ab,kw OR lipidaemia:ti,ab,kw OR lipidemia:ti,ab,kw) AND ('hydroxymethylglutaryl coenzyme a reductase \\
& inhibitor'/exp OR 'hydroxymethylglutaryl coenzyme a reductase inhibitor':ti,ab,kw OR 'hydroxymethylglutaryl-coa inhibitors':ti,ab,kw OR \\
'hydroxymethylglutaryl-coenzyme a inhibitors':ti,ab,kw OR 'hmg coa reductase inhibitor':ti,ab,kw OR 'hmg coenzyme a reductase inhibitor': & ti,ab,kw OR 'hmg coa reductase inhibitors':ti,ab,kw OR 'hydroxymethylglutaryl coa reductase inhibitors':ti,ab,kw OR 'hydroxymethylglutaryl- \\
& coa reductase inhibitors':ti,ab,kw OR statin:ti,ab,kw OR statins:ti,ab,kw OR vastatin:ti,ab,kw)
\end{tabular}

limited inclusion to randomized controlled trials (RCTs). Studies were excluded for key questions if full text was not available.

Search results were uploaded into systematic review software (DistillerSR, Ottawa, Canada). In the first round of screening, abstracts and titles were evaluated for inclusion. Following abstract screening, eligibility was assessed through full-text screening. Prior to both abstract and full text screening, reviewers underwent training to ensure a basic understanding of the background of the field and purpose of the review as well as comprehension of the inclusion and exclusion criteria. The initial 20 abstracts were reviewed independently and then discussed as a group. Eligibility at both levels (abstract and full-text) was assessed independently and in duplicate (L.K.J., S.T., L.R.F., and C.G.). Disagreements at the level of abstract and full text screening were resolved by consensus. If consensus could not be achieved between the two reviewers, a third reviewer arbitrated (M.R.G., T.W., or T.S.).

\section{Data collection}

The following characteristics were extracted from included studies: first author, year of publication, location, age of patient population (adult vs. child), study design, implementation strategies, inclusion and exclusion criteria, and any of the following outcomes: statin prescribing or use, statin adherence, or LDL-C measurements.
Key question 1: what implementation strategies have been used to promote the uptake of statins?

We first summarized and described the populations, interventions, comparisons, and outcomes presented for all studies that reported at least one implementation strategy that could be mapped to the ERIC compilation. The ERIC compilation of nine implementation strategies categories (73 total strategies) was applied to each of the interventions to (1) count the total number of strategies and (2) describe how complete each implemented strategy was defined. One study team member, who was an author on the original ERIC compilation, ensured validity of the categories selected (T.W.) [14].

\section{Key question 2: how completely are the implementation strategies utilized reported in studies designed to promote statin uptake?}

Based on guidance from proctor and colleagues, we assessed the degree to which each strategy was completely reported including actor, action, action target, temporality, dose, implementation outcome affected, and justification (Table 2) [11].

Key question 3: which implementation strategy, or combination of strategies, is (are) the most effective at promoting the uptake of statins?

When present, we extracted data related to statin prescribing, statin adherence, and LDL-C reported from included RCTs. All outcomes were collected at

Table 2 Summary of the implementation strategies' defining characteristics

\begin{tabular}{ll}
\hline Characteristics & Definition \\
\hline Actor & Identify who enacts the strategy \\
Action & Specific actions, steps, or processes that need to be enacted \\
Action Target & 1) Specify targets according to conceptual models of implementation \\
& 2) Identify unit of analysis for measuring implementation outcomes \\
Temporality & Specify when the strategy is used \\
Dose & Specify dosage of implementation strategy \\
Implementation outcome affected & Identify and measure the implementation outcome(s) that are affected by each strategy \\
Justification & Justification for choice of implementation strategies
\end{tabular}

Characteristics and definitions were utilized from Proctor 2013. The justification definition was adjusted to reflect an argument for the implementation strategy by noting an implementation science framework or guidance and not an evidence-base for the intervention 
intervention completion. Statin prescribing or use included all orders for statin medications. Statin adherence included only objective measures of adherence by either medication possession ratio (MPR) or proportion of days covered (PDC) [18]. MPR or PDC were captured as a percentage or attainment of greater than $80 \%$ adherence. LDL-C levels were recorded as LDL-C measured or achievement of an LDL-C target.

\section{Risk of bias assessment}

The Cochrane Collaboration's risk of bias tool version 2 to evaluate methodological quality of studies included in the meta-analysis for key question 3 [19]. The risk of bias in included studies was assessed in duplicate by two reviewers (L.K.J. and L.R.F.) working independently. Any disagreements were resolved by consensus; if consensus was unable to be achieved, a third reviewer arbitrated (M.R.G.).

\section{Statistical analysis}

Standardized mean differences (SMDs) with corresponding 95\% CIs were estimated for continuous outcomes, and odds ratios (ORs) and 95\% confidence intervals (CIs) were calculated for binary outcomes from included studies. Publication bias was evaluated by Egger's test [20]. Variability between included studies was assessed by heterogeneity tests using $I^{2}$ statistic [21]. If overall results showed significant heterogeneity, potential sources of heterogeneity were explored by subgroup analysis. All analyses were conducted using RStudio (Version 1.0.136) using the "Meta" and "Metafor" package.

\section{Results}

Description of study selection

We initially identified 65,118 studies. After removing duplicates, we identified 38,585 unique citations (Fig. 1). Through abstract and title screening, 208 reports were

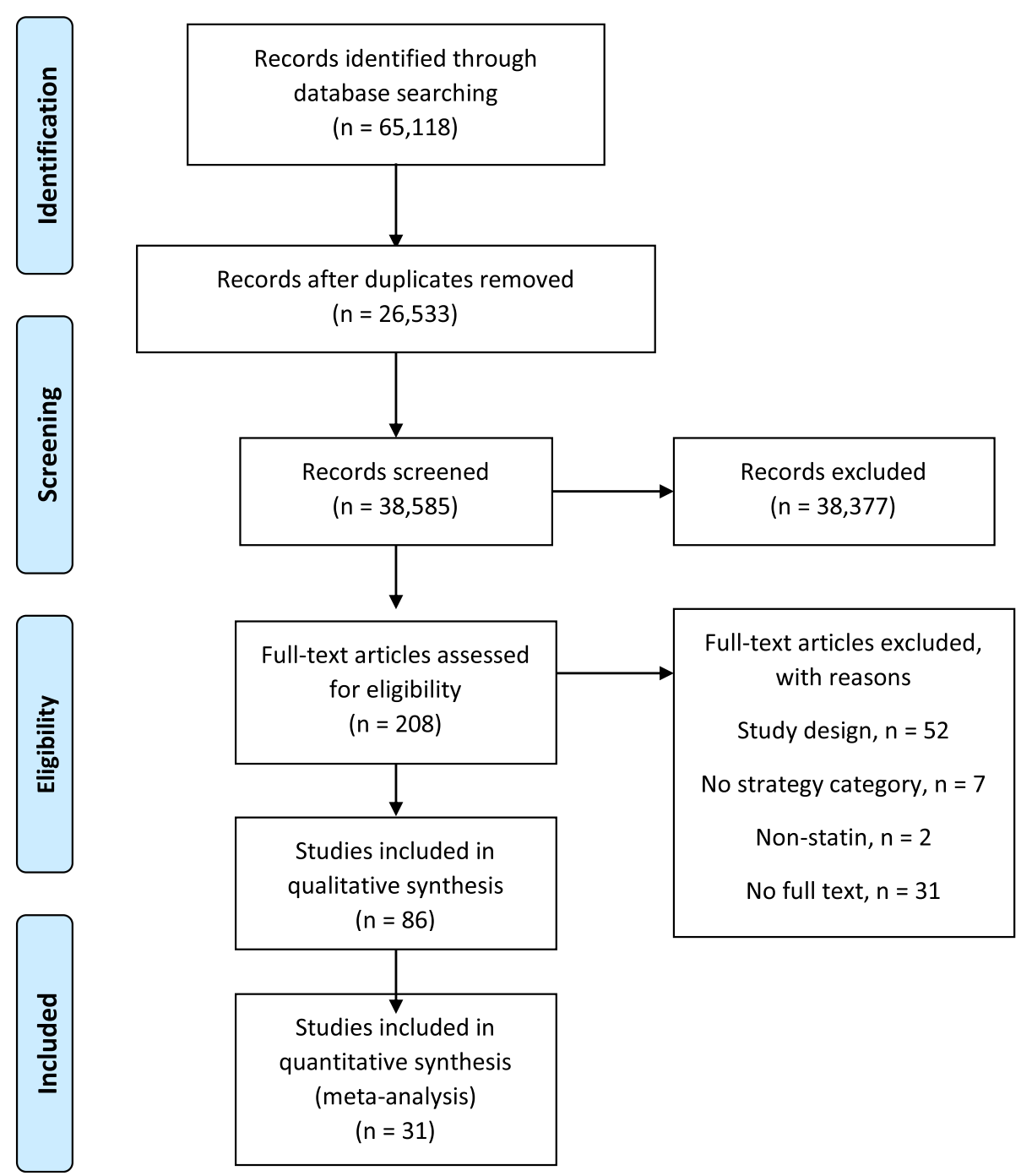

Fig. 1 PRISMA flow diagram 
Table 3 Study demographics

\begin{tabular}{|c|c|c|c|c|c|c|}
\hline Year & $\begin{array}{l}\text { Author last } \\
\text { name }\end{array}$ & Location & Population & Study design & Outcomes measured & $\begin{array}{l}\text { Included in meta- } \\
\text { analysis }\end{array}$ \\
\hline 1996 & Schectman [34] & United States & Adult & RCT & LDL-C, Statin Adherence & $\checkmark$ \\
\hline 1997 & Bogden [65] & United States & Adult & $\mathrm{RCT}$ & LDL-C & $\checkmark$ \\
\hline 2000 & Nordmann [37] & Switzerland & Adult & $\mathrm{RCT}$ & Statin Prescribing & $\checkmark$ \\
\hline 2000 & Nguyen [38] & France & Adult & $\mathrm{RCT}$ & LDL-C & $\checkmark$ \\
\hline 2000 & Faulkner [71] & United States & $\begin{array}{l}\text { Adult and } \\
\text { Child }\end{array}$ & $\mathrm{RCT}$ & LDL-C & $\checkmark$ \\
\hline 2005 & Rachmani [36] & Israel & Adult & $\mathrm{RCT}$ & LDL-C, Statin Prescribing & $\checkmark$ \\
\hline 2006 & Lester [41] & United States & Adult & $\mathrm{RCT}$ & LDL-C & $\checkmark$ \\
\hline 2006 & Lee [78] & United States & Adult & $\mathrm{RCT}$ & LDL-C & $\checkmark$ \\
\hline 2007 & Khanal [77] & United States & Adult & RCT & LDL-C, Statin Prescribing & $\checkmark$ \\
\hline 2008 & Riesen [35] & Switzerland & Adult & $\mathrm{RCT}$ & LDL-C & $\checkmark$ \\
\hline 2009 & Stacy [31] & United States & Adult & $\mathrm{RCT}$ & Statin Adherence & $\checkmark$ \\
\hline 2009 & Willich [89] & Germany & Adult & $\mathrm{RCT}$ & LDL-C & $\checkmark$ \\
\hline 2009 & McAlister [101] & Canada & Adult & RCT & LDL-C, Statin Prescribing & $\checkmark$ \\
\hline 2010 & Webster [23] & Australia & Adult & $\mathrm{RCT}$ & LDL-C & $\checkmark$ \\
\hline 2010 & Villeneuve [103] & Canada & Adult & RCT & LDL-C, Statin Prescribing & $\checkmark$ \\
\hline 2012 & Nieuwkerk [86] & Netherlands & Adult & $\mathrm{RCT}$ & LDL-C & $\checkmark$ \\
\hline 2013 & Zamora [12] & Spain & Adult & $\mathrm{RCT}$ & LDL-C & $\checkmark$ \\
\hline 2013 & Kooy [42] & Netherlands & Adult & $\mathrm{RCT}$ & Statin Adherence & $\checkmark$ \\
\hline 2013 & Kardas [43] & Poland & Adult & $\mathrm{RCT}$ & Statin Adherence & $\checkmark$ \\
\hline 2013 & Goswami [72] & United States & Adult & $\mathrm{RCT}$ & Statin Adherence & $\checkmark$ \\
\hline 2014 & McAlister [83] & Canada & Adult & $\mathrm{RCT}$ & LDL-C, Statin Prescribing & $\checkmark$ \\
\hline 2014 & Lowrie [100] & $\begin{array}{l}\text { United } \\
\text { Kingdom }\end{array}$ & Adult & $\mathrm{RCT}$ & Statin Prescribing & $\checkmark$ \\
\hline 2015 & Mols [54] & Denmark & Adult & $\mathrm{RCT}$ & LDL-C & $\checkmark$ \\
\hline 2015 & Asch [13] & United States & Adult & RCT & LDL-C & $\checkmark$ \\
\hline 2015 & Patel [82] & Australia & Adult & RCT & LDL-C & $\checkmark$ \\
\hline 2016 & Jakobsson [44] & Sweden & Adult & $\mathrm{RCT}$ & LDL-C, Statin Prescribing & $\checkmark$ \\
\hline 2016 & Damush [79] & United States & Adult & $\mathrm{RCT}$ & Statin Adherence & $\checkmark$ \\
\hline 2018 & Choudhry [76] & United States & Adult & RCT & LDL-C, Statin Adherence & $\checkmark$ \\
\hline 2018 & Mehrpooya [80] & Iran & Adult & $\mathrm{RCT}$ & LDL-C & $\checkmark$ \\
\hline 2018 & Martinez [81] & Spain & Adult & RCT & LDL-C & $\checkmark$ \\
\hline 2018 & Osborn [104] & $\begin{array}{l}\text { United } \\
\text { Kingdom }\end{array}$ & Adult & $\mathrm{RCT}$ & LDL-C, Statin Prescribing & $\checkmark$ \\
\hline 1996 & Lindholm [39] & Sweden & Adult & $\mathrm{RCT}$ & LDL-C & \\
\hline 2003 & Sebregts [99] & Netherlands & $\begin{array}{l}\text { Adult and } \\
\text { Child }\end{array}$ & $\mathrm{RCT}$ & LDL-C & \\
\hline 2007 & Choe [95] & United States & Adult & $\mathrm{RCT}$ & LDL-C, Statin Adherence & \\
\hline 2008 & Hung [90] & Taiwan & Adult & $\mathrm{RCT}$ & LDL-C, Statin Prescribing & \\
\hline 2010 & $\begin{array}{l}\text { Bhattacharyya } \\
\text { [62] }\end{array}$ & Canada & Adult & $\mathrm{RCT}$ & LDL-C, Statin Prescribing & \\
\hline 2013 & Dresser [55] & Canada & Adult & $\mathrm{RCT}$ & LDL-C & \\
\hline 2013 & Brath [60] & Austria & Adult & $\mathrm{RCT}$ & LDL-C, Statin Adherence & \\
\hline 2013 & Derose [85] & United States & Adult & $\mathrm{RCT}$ & Statin Adherence & \\
\hline 2005 & Straka [28] & United States & Adult & $\begin{array}{l}\text { Nonrandomized Clinical } \\
\text { Trial }\end{array}$ & LDL-C & \\
\hline
\end{tabular}


Table 3 Study demographics (Continued)

\begin{tabular}{|c|c|c|c|c|c|c|}
\hline Year & $\begin{array}{l}\text { Author last } \\
\text { name }\end{array}$ & Location & Population & Study design & Outcomes measured & $\begin{array}{l}\text { Included in meta- } \\
\text { analysis }\end{array}$ \\
\hline 2005 & Paulos [105] & Chile & Adult & $\mathrm{RCT}$ & LDL-C, Statin Adherence & \\
\hline 2006 & Vrijens [24] & Belgium & Adult & $\mathrm{RCT}$ & Statin Adherence & \\
\hline 2015 & Persell [102] & United States & Adult & $\mathrm{RCT}$ & LDL-C, Statin Prescribing & \\
\hline 2017 & Bosworth [61] & United States & Adult & $\mathrm{RCT}$ & LDL-C, Statin Adherence & \\
\hline 2018 & Etxeberria [53] & Spain & $\begin{array}{l}\text { Adult and } \\
\text { Child }\end{array}$ & $\mathrm{RCT}$ & Statin Prescribing & \\
\hline 1995 & Shaffer [94] & United States & Adult & Observational & LDL-C & \\
\hline 1997 & Shibley [32] & United States & Adult & Observational & LDL-C & \\
\hline 1999 & Schwed [33] & Switzerland & Adult & Observational & LDL-C, Statin Adherence & \\
\hline 2000 & Robinson [92] & United States & Adult & Observational & LDL-C, Statin Prescribing & \\
\hline 2000 & Birtcher [93] & United States & Adult & Observational & Statin Prescribing & \\
\hline 2001 & Ford [52] & $\begin{array}{l}\text { United } \\
\text { Kingdom }\end{array}$ & Adult & Observational & Statin Prescribing & \\
\hline 2002 & Viola [25] & United States & Adult & Observational & LDL-C, Statin Prescribing & \\
\hline 2002 & Geber [50] & United States & Adult & Observational & LDL-C & \\
\hline 2002 & Gavish [51] & Israel & Adult & Observational & LDL-C, Statin Adherence & \\
\hline 2002 & Hilleman [70] & United States & Adult & Observational & LDL-C; Statin Prescribing & \\
\hline 2003 & Truppo [27] & United States & Adult & Observational & LDL-C; Statin Adherence & \\
\hline 2003 & Ryan [98] & United States & Adult & Observational & LDL-C; Statin Prescribing & \\
\hline 2004 & Hilleman [45] & United States & $\begin{array}{l}\text { Adult and } \\
\text { Child }\end{array}$ & Observational & LDL-C & \\
\hline 2004 & de Velasco [56] & Spain & Adult & Observational & LDL-C, Statin Prescribing & \\
\hline 2004 & Lappé [69] & United States & Adult & Observational & Statin Prescribing & \\
\hline 2005 & Harats [47] & Israel & Adult & Observational & LDL-C & \\
\hline 2005 & Bassa [63] & Spain & Adult & Observational & LDL-C & \\
\hline 2005 & Brady [91] & $\begin{array}{l}\text { United } \\
\text { Kingdom }\end{array}$ & Adult & Observational & Statin Prescribing & \\
\hline 2005 & McLeod [96] & $\begin{array}{l}\text { United } \\
\text { Kingdom }\end{array}$ & Adult & Observational & Statin Adherence & \\
\hline 2005 & Rabinowitz [97] & Israel & Adult & Observational & LDL-C & \\
\hline 2006 & de Lusignan [57] & $\begin{array}{l}\text { United } \\
\text { Kingdom }\end{array}$ & $\begin{array}{l}\text { Adult and } \\
\text { Child }\end{array}$ & Observational & Statin Prescribing & \\
\hline 2006 & Rehring [66] & United States & Adult & Observational & LDL-C & \\
\hline 2007 & Goldberg [48] & United States & Adult & Observational & LDL-C & \\
\hline 2008 & Stockl [29] & United States & Adult & Observational & $\begin{array}{l}\text { Statin Prescribing, Statin } \\
\text { Adherence }\end{array}$ & \\
\hline 2008 & Hatfield [67] & $\begin{array}{l}\text { United } \\
\text { Kingdom }\end{array}$ & Adult & Observational & LDL-C, Statin Adherence & \\
\hline 2008 & Coodley [88] & United States & Both & Observational & LDL-C & \\
\hline 2009 & Stephenson [30] & United States & $\begin{array}{l}\text { Adult and } \\
\text { Child }\end{array}$ & Observational & LDL-C & \\
\hline 2009 & Lima [40] & Brazil & Adult & Observational & LDL-C & \\
\hline 2009 & Casebeer [59] & United States & Adult & Observational & Statin Adherence & \\
\hline 2010 & Chen [75] & Taiwan & Adult & Observational & LDL-C & \\
\hline 2011 & Gitt [49] & Germany & Adult & Observational & LDL-C & \\
\hline 2011 & Chung [58] & Hong Kong & Adult & Observational & LDL-C & \\
\hline 2011 & Schmittdiel [87] & United States & Adult & Observational & LDL-C & \\
\hline
\end{tabular}


Table 3 Study demographics (Continued)

\begin{tabular}{|c|c|c|c|c|c|c|}
\hline Year & $\begin{array}{l}\text { Author last } \\
\text { name }\end{array}$ & Location & Population & Study design & Outcomes measured & $\begin{array}{l}\text { Included in meta- } \\
\text { analysis }\end{array}$ \\
\hline 2012 & Aziz [68] & United States & Adult & Observational & LDL-C, Statin Prescribing & \\
\hline 2012 & Farley [74] & United States & Adult & Observational & Statin Adherence & \\
\hline 2014 & Clark [73] & United States & $\begin{array}{l}\text { Adult and } \\
\text { Child }\end{array}$ & Observational & Statin Adherence & \\
\hline 2014 & Shoulders [84] & United States & Adult & Observational & LDL-C, Statin Prescribing & \\
\hline 2015 & Vinker [26] & Israel & Adult & Observational & LDL-C, Statin Prescribing & \\
\hline 2016 & Harrison [46] & United States & Adult & Observational & LDL-C, Statin Adherence & \\
\hline 2017 & Andrews [64] & United States & Adult & Observational & Statin Adherence & \\
\hline 2018 & Weng [22] & $\begin{array}{l}\text { United } \\
\text { Kingdom }\end{array}$ & Adult & Observational & LDL-C, Statin Prescribing & \\
\hline
\end{tabular}

identified for full-text review. During full-text review, 86 were selected for inclusion [12, 13, 22-105]. A complete list of excluded full-text studies with rationale for exclusion is available in Additional file 1: Appendix 2.

\section{Description of studies}

Table 3 describes the included studies (more details are included in Additional file 1: Appendix 3). Almost all the implementation strategies targeted adults (two studies included pediatric patients), half were implemented in the USA, and almost all were conducted in individuals with hypercholesterolemia (two studies were conducted in individuals with familial hypercholesterolemia).

\section{Implementation strategies}

All implementation strategies except "provide interactive assistance" were used (Table 4). A total of 258 uses of strategies were identified across 86 studies. On average, each study utilized three strategies (SD 2.2, range 1-13). The most utilized strategies were "train and educate the stakeholders" (studies utilized strategies in this grouping 79 times), "support clinicians" (68), and "engage consumers" (47). The most utilized individual strategies were "intervene with patients and consumers to enhance uptake and adherence" (41), and "distribute educational materials" (41) (Additional file 1: Appendix 4). Implementation strategies often did not include key defining characteristics: temporality was reported $59 \%$ of the time, dose $52 \%$, affected outcome $9 \%$, and justification 6\% (Table 2 provides a summary and Additional file 1: Appendix 5 provides a more detailed version).

\section{Meta-analysis}

Due to the large heterogeneity between studies, effectiveness outcomes (statin prescribing, statin adherence, and LDL-C) were only extracted from RCTs. Thirty-one trials reported at least one of the three outcomes of interest. The implementation strategies examined demonstrated: significantly reduced LDL-C (LDL-C reduction: SMD $-0.17,95 \% \mathrm{CI}-0.27$ to $-0.07, p=0.0006$; met LDL-C target: OR $1.33,95 \%$ CI 1.13 to $1.58, p=$ 0.0008 ) (Fig. 2), increased rates of statin prescribing (OR $2.21,95 \%$ CI 1.60 to $3.06, p<0.0001$ ) (Fig. 3), and improved statin adherence (PDC/MPR: SMD 0.13, 95\% CI 0.06 to $0.19 ; p=0.0002 ; \geq 80 \%$ PDC/MPR: OR $1.30,95 \%$ CI 1.04 to $1.63, p=0.023$ ) (Fig. 4). There was inconsistency across trials based on the outcome measured; statin prescribing $\left(I^{2}=73 \%\right)$, statin adherence $\left(I^{2}=0 \%\right)$, and LDL-C $\left(I^{2}=79 \%\right.$ (LDL-C reduction) and 76\% (met LDL$\mathrm{C}$ targets)). Publication bias using the Egger's test

Table 4 Summary of implementation strategies by strategy category

\begin{tabular}{llll}
\hline Strategy category & Strategies used per category & Total count within category & Meta-analysis total count within category \\
\hline Use evaluative and iterative strategies & $80 \%(8 / 10)$ & 33 & 9 \\
Support clinicians & $80 \%(4 / 5)$ & 68 & 20 \\
Adapt and tailor to the context & $75 \%(3 / 4)$ & 4 & 2 \\
Engage consumers & $60 \%(3 / 5)$ & 47 & 24 \\
Train and educate the stakeholders & $55 \%(6 / 11)$ & 80 & 2 \\
Change infrastructure & $50 \%(4 / 8)$ & 9 & 5 \\
Develop stakeholder relationships & $47 \%(8 / 17)$ & 11 & 2 \\
Utilize financial strategies & $22 \%(2 / 9)$ & 6 & 0 \\
Provide interactive assistance & $0 \%(0 / 4)$ & 0 & \\
\hline
\end{tabular}


a)

Study
Zamora 2013
Schectman 1996
Riesen 2008
Nguyen 2000
Jakobsson 2016
Mols 2015
Bogden 1997
Faulkner 2000
Asch 2015
Asch 2015
Asch 2015
Khanal 2007
McAlister 2014
Willich 2009
McAlister 2009
Villeneuve 2010

\section{Experimental Control} Events Total Events Total

$\begin{array}{rrrr}25 & 33 & 21 & 44 \\ 67 & 103 & 47 & 105 \\ 286 & 471 & 358 & 531 \\ 1067 & 1407 & 1083 & 1443 \\ 66 & 101 & 36 & 100 \\ 64 & 94 & 68 & 95 \\ 21 & 47 & 10 & 47 \\ 8 & 15 & 2 & 15 \\ 144 & 358 & 132 & 366 \\ 174 & 433 & 132 & 366 \\ 170 & 346 & 132 & 366 \\ 479 & 617 & 479 & 616 \\ 74 & 143 & 46 & 136 \\ 2231 & 4042 & 2152 & 4022 \\ 129 & 323 & 120 & 314 \\ 87 & 108 & 86 & 117\end{array}$

Random effects model 8641 Heterogeneity: $I^{2}=76 \%, \tau^{2}=0.0673, p<0.01$

8683

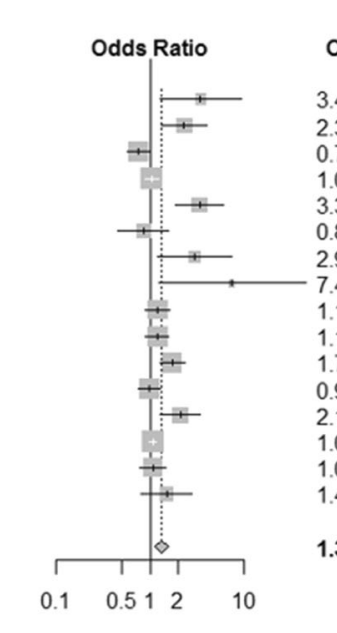

b)

Study

Schectman 1996 Nguyen 2000 Faulkner 2000 Rachmani 2005 Lester 2005

Lee 2006

Khanal 2007

McAlister 2009
Webster 2010

Nieuwkerk 2012

Nieuwkerk 2012

Zamora 2013

McAlister 2014

Mols 2015

Patel 2015

Mehrpooya 2018

Osborn 2018

Random effects model 5390

Heterogeneity. $I^{2}=79 \%, \tau^{2}=0.0297, p<0.01$

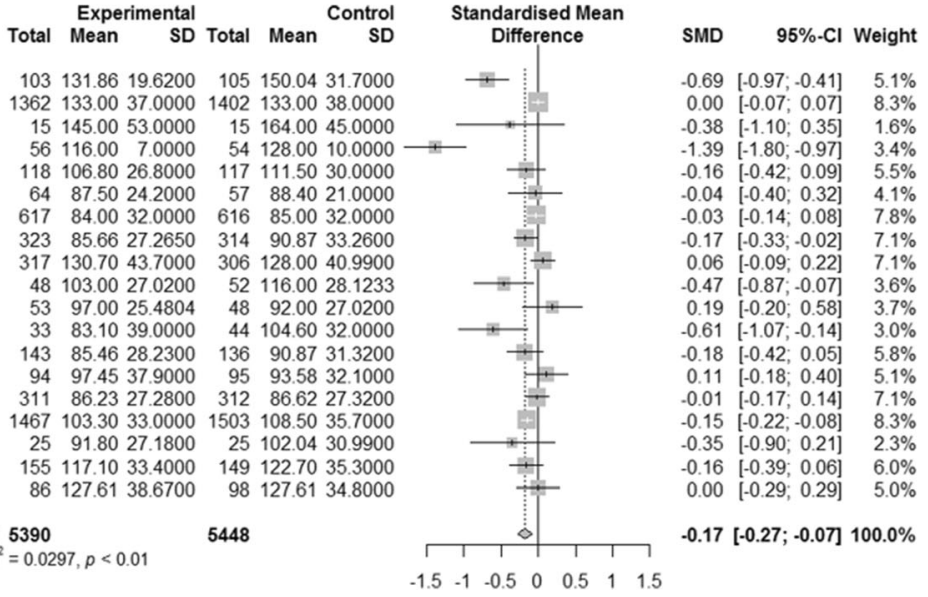

$\begin{array}{lllllll}-1.5 & -1 & -0.5 & 0 & 0.5 & 1 & 1.5\end{array}$

\section{OR $\quad 95 \%-\mathrm{Cl}$ Weight}

3.42 [1.27; 9.23] 2.3\%

$2.30[1.31 ; 4.02] \quad 5.0 \%$

$0.75[0.58 ; 0.97] \quad 8.7 \%$

$1.04[0.88 ; 1.24] \quad 9.8 \%$

$3.35[1.88 ; 5.98] \quad 4.8 \%$

$0.85[0.45 ; 1.58] \quad 4.4 \%$

$2.99[1.21 ; 7.39] \quad 2.6 \%$

$7.43[1.23 ; 45.01] \quad 0.8 \%$

[0.88; 1.61] $8.1 \%$

$19[0.89 ; 1.59] \quad 8.3 \%$

$71[1.27 ; 2.31] \quad 8.1 \%$

$0.99[0.76 ; 1.30] \quad 8.6 \%$

$10[1.29 ; 3.40] \quad 5.7 \%$

$07 \quad 0.98 ; 1.17] \quad 10.6 \%$

$07[0.78 ; 1.48] \quad 7.9 \%$

$1.49[0.80 ; 2.80] \quad 4.3 \%$

$1.33[1.13 ; 1.58] 100.0 \%$

Fig. 2 Forest plot of implementation strategies' impact on LDL-C compared to control. a Achievement of target LDL-C. b Standardized mean difference in LDL-C

\begin{tabular}{|c|c|c|c|c|}
\hline Study & $\begin{array}{l}\text { Experim } \\
\text { Events }\end{array}$ & $\begin{array}{r}\text { nental } \\
\text { Total }\end{array}$ & Events & $\begin{array}{l}\text { Ontrol } \\
\text { Total }\end{array}$ \\
\hline Zamora 2013 & 25 & 33 & 15 & \\
\hline Schectman 1996 & 96 & 103 & 65 & \\
\hline Rachmani 2005 & 56 & 56 & 36 & \\
\hline Nordmann 2000 & 26 & 30 & 13 & \\
\hline Jakobsson 2016 & 97 & 101 & 91 & 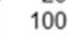 \\
\hline Khanal 2007 & 580 & 617 & 543 & \\
\hline McAlister 2014 & 85 & 143 & 77 & \\
\hline Lowrie 2014 & 3682 & 4234 & 2509 & 335 \\
\hline McAlister 2009 & 277 & 323 & 258 & \\
\hline Villeneuve 2010 & 92 & 108 & 95 & 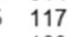 \\
\hline Osborn 2018 & 38 & 144 & 31 & \\
\hline
\end{tabular}

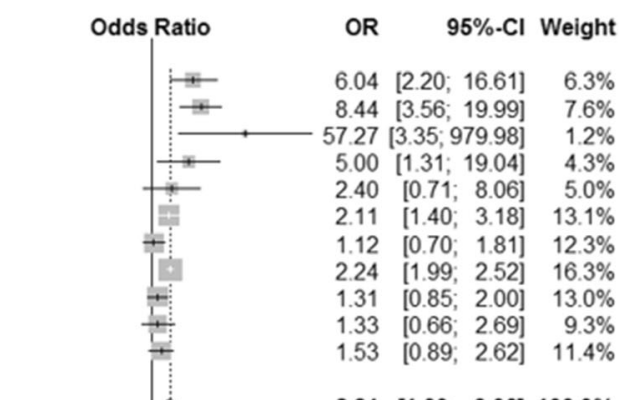

$2.21[1.60 ; 3.06] 100.0 \%$

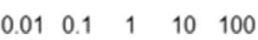

Fig. 3 Forest plot of implementation strategies' impact on statin prescribing compared to control 
a)

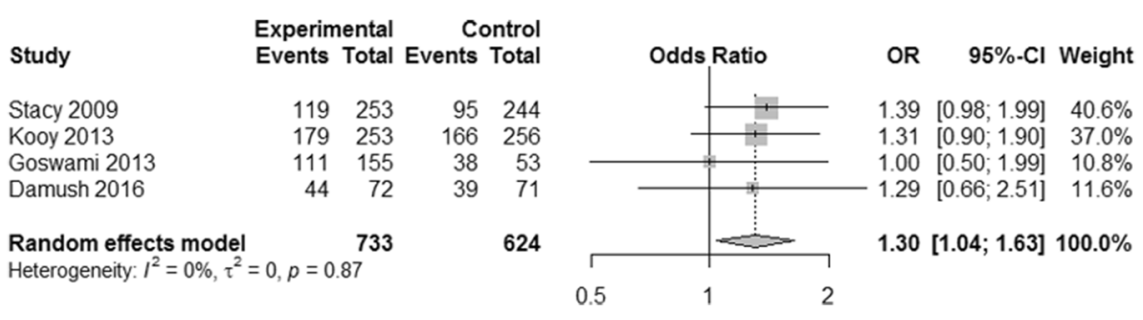

b)

\begin{tabular}{|c|c|c|c|c|}
\hline \multirow[b]{2}{*}{ Study } & \multicolumn{2}{|r|}{ Experimental } & \multicolumn{2}{|r|}{ Control } \\
\hline & Total & Mean & Total & Mean \\
\hline Kardas 2013 & 107 & 0.950 .5370 & 89 & $0.82 \quad 0.3100$ \\
\hline Goswami 2013 & 155 & 0.820 .2300 & 53 & 0.810 .2400 \\
\hline Choudhry 2018 & 1467 & 0.480 .3370 & 1503 & $0.44 \quad 0.3360$ \\
\hline Damush 2016 & 72 & 0.770 .2600 & 71 & 0.750 .2600 \\
\hline $\begin{array}{l}\text { Random effec } \\
\text { Heterogeneity: } I^{2}\end{array}$ & $\begin{array}{l}1801 \\
0, p\end{array}$ & 59 & 1716 & \\
\hline
\end{tabular}

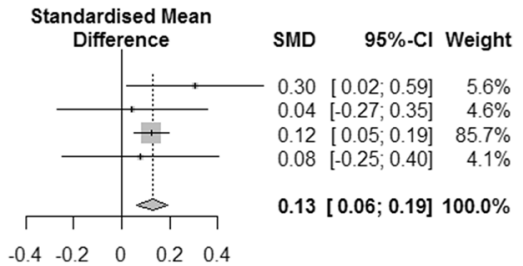

Fig. 4 Forest plot of implementation strategies' impact on statin adherence compared to control. a Medication possession ratio or portion of days covered $>80 \%$. b Standardized mean difference in medication possession ratio or portion of days covered

indicated no publication bias for statin prescribing $(p=$ $0.63)$, statin adherence ( $p=0.83$ for SMD, $p=0.22$ for $\mathrm{OR})$, and potential publication bias for LDL-C $(p=0.08$ for SMD, $p=0.01$ for OR).

Although subgroup analyses were conducted for statin prescribing and LDL-C, there were not enough studies to conduct a subgroup analysis for statin adherence (Table 5). We identified a significant difference among studies published in 2013 or later for LDL-C measured as a binary outcome (OR 1.62, 95\% CI1.19-2.19, $p=0.05$ ). We also found a significant effect on LDL-C measured as a continuous variable when more than 2 implementation strategies were utilized (SMD - $0.3895 \% \mathrm{CI}-0.67 ;-0.09, p=0.05$ ). There was no significant effect in the between country analysis.

Most studies were found to be at a low risk of bias (Fig. 5 and Additional file 1: Appendix 6); therefore, we did not conduct subgroup analyses based on the risk of bias.

\section{Discussion}

\section{Our findings}

In this review of implementation strategies regarding uptake of statins in hypercholesterolemia, we found that 38 different strategies were utilized to lower LDL-C, improve

Table 5 Subgroup analyses

\begin{tabular}{|c|c|c|c|}
\hline Study subgroup (number of studies) & Subgroup & Comparison group & $P$ value for interaction \\
\hline \multicolumn{4}{|l|}{ Odds ratio, $(95 \% \mathrm{Cl})$} \\
\hline \multicolumn{4}{|l|}{ Statin prescribing (11) } \\
\hline More than 2 implementation strategies (6) & $2.19(1.32-3.63)$ & $2.40(1.43-4.06)$ & 0.80 \\
\hline Study published in 2013 or later (5) & $1.97(1.29-3.01)$ & $2.84(1.41-5.74)$ & 0.36 \\
\hline Conducted in the United States (2) & $4.00(1.03-15.50)$ & $1.95(1.33-2.84)$ & 0.32 \\
\hline \multicolumn{4}{|l|}{$L D L-C(14)$} \\
\hline More than 2 implementation strategies (4) & $1.53(1.23-1.90)$ & $1.20(0.97-1.48)$ & 0.12 \\
\hline Study published 2013 or later (5) & $1.62(1.19-2.19)$ & $1.13(0.95-1.35)$ & 0.05 \\
\hline Conducted in the United States (5) & $1.48(1.12-1.95)$ & $1.29(1.03-1.61)$ & 0.35 \\
\hline \multicolumn{4}{|l|}{ Standardized mean difference, (95\% Cl) } \\
\hline \multicolumn{4}{|l|}{$L D L-C(17)$} \\
\hline More than 2 implementation strategies (6) & $-0.38(-0.67 ;-0.09)$ & $-0.07(-0.15 ;-0.01)$ & 0.05 \\
\hline Study published in 2013 or later (8) & $-0.12(-0.21 ; \vee 0.02)$ & $-0.23(-0.39 ;-0.07)$ & 0.24 \\
\hline Conducted in the United States (6) & $-0.20(-0.36 ;-0.04)$ & $-0.17(-0.31 ;-0.03)$ & 0.79 \\
\hline
\end{tabular}

Statin adherence was excluded because there were not enough studies to make a comparison 


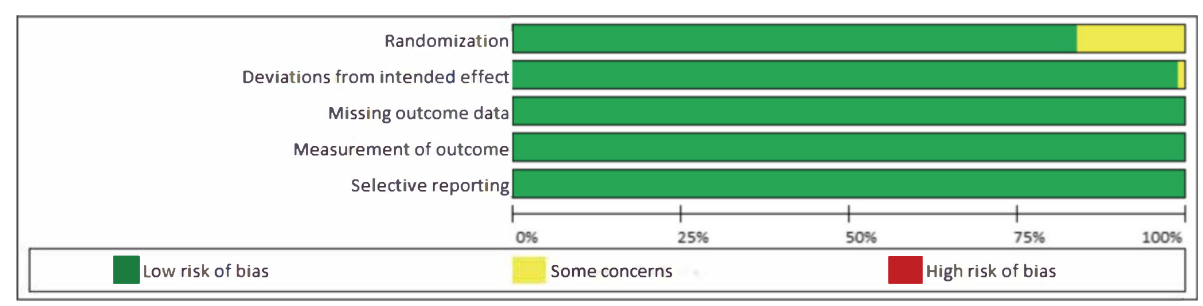

Fig. 5 Risk of bias of RCTs included in the meta-analyses

statin prescribing, and promote adherence. However, strategy components were not well defined and there was not a single strategy or group of strategies that demonstrated superior impact compared to others. Consistent with management of other diseases and conditions and literature from implementation science [106], we found evidence to support the use of multiple concurrent strategies; the use of three or more implementation strategies was associated with a greater reduction in LDL-C. We also found that studies published after 2012 had, on average, greater reductions in LDL-C through the use of the reported implementation strategies. While it cannot be definitely attributed, this could result from a better understanding of which strategies work best or could reflect a switch toward the utilization of high dose statin therapy. There was no difference in outcomes based on country where the study was conducted.

An important limitation of the many strategies described was incomplete definitions, limiting generalizability to other settings. Often, we were able to discern the actor, action, and action target but were unable to determine temporality, dose, implementation outcome affected, or justification. Without clear reporting of these factors, we are unable to interpret when these strategies should be used (temporality), how often (dosage), how the success of a specific strategy is measured (implementation outcomes affected), or when to justify the choice of a particular strategy (justification) to influence clinical practice. While the interventions appeared to be effective at increasing the utilization of statins and reducing LDL-C overall, the variable nature of the interventions studied and outcomes examined, the effectiveness of any specific strategy or set of strategies was unclear.

In addition, one category of strategies, "provide interactive assistance," was not utilized in any of the studies included in the analysis. Among the strategies that were used, many were used in combination, but specific combinations were not used frequently enough to permit reliable subgroup analysis.

\section{Comparison with other studies}

In the field of implementation science, there has recently been a desire to improve specification of implementation strategies utilized in practice and to develop standard language and definitions for reporting these implementation strategies $[11,14,107]$. This trend has led to the development of two implementation strategy taxonomies: the ERIC compilation [14], used in this study, and the Effective Practise and Organization of Care (EPOC) taxonomy [17]. Use of these taxonomies has allowed for consistent language in reporting implementation strategies and development of tailored compilations of strategies specific to certain disease states [108, 109]. Other systematic reviews of implementation strategies in other fields (i.e., intensive care setting and oral health) have found improved outcomes when multiple implementation strategies are used but have not been able to identify the groups of strategies most likely to produce the most favorable outcomes [110-112].

An investigation of enablers and barriers to treatment adherence in familial hypercholesterolemia found seven enablers for patients that could be used to develop new interventions and matched to implementation strategies we identified in our study [113]. These enablers were "other family members following treatment regime," "commencement of treatment from a young age," "parental responsibility to care for children," "confidence in ability to successfully self-manage their condition," "receiving formal diagnosis of familial hypercholesterolemia," "practical resources and support for following lifestyle treatment," and a "positive relationship with healthcare professionals" [113]. By linking the two most frequently used strategies identified in our systematic review "intervene with patients and consumers to enhance uptake and adherence" and "distribute educational materials," with the enablers identified above, effective implementations strategies for statin utilization can be designed.

The sustainability of interventions to promote the uptake of guidelines when intervening at the clinician level has been limited in a variety of settings [114-116]. Specifically, in cardiovascular disease, a systematic review of interventions to improve uptake of heart failure medications saw an increase in guideline uptake but not improvement in clinical outcomes [117]. Similar findings have been found in hypertension [118]. However, the success of these interventions have been limited. 


\section{Limitations and strengths}

Our review is the first to comprehensively map the strategies used to increase utilization of statins among persons with hypercholesterolemia to the ERIC compilation. We chose to use ERIC due to a perceived better fit over alternatives (i.e. EPOC); however, we identified 7 studies (out of 208 identified) which could not be mapped to ERIC, exclusion of which could lead to missing important strategies. Other strengths include utilization of a medical librarian to conduct the search, searching of multiple databases which covered parts of the gray literature, and utilizing trained reviewers. Finally, we limited our search to studies in English with full-texts available. Thus, we may have missed studies not published in English or published in the gray literature (e.g., only conference abstract available in published literature) and be at risk for language bias [119] or publication bias [120]. While the Egger's test suggested possible publication bias, we think that the risk of this is low due to our comprehensive search strategy. Further, while language bias is a possibility [119], few studies were excluded based on language so any potential impact is likely to be small.

\section{Suggestions for future research}

Consistent strategies for reporting LDL-C would significantly improve the ability to assess efficacy of an intervention. Some studies used arbitrary cut-offs for LDL-C, some used absolute values, and others used thresholds published in cholesterol guidelines [121]. This led to difficulty in aggregating data across studies. Future studies should report absolute values of LDL-C to facilitate meta-analyses directed at change of LDL-C with intervention. Generating a core outcome set for trials in hypercholesterolemia would facilitate meta-analyses and ensure all relevant outcomes are consistently measured [122]. Ideally, these studies should be registered and included in a meta-analysis in a prospective manner [123].

Clarity in the terminology, definition, and description of implementation strategies by researchers would help translation and replication of efforts. Completely reporting implementation strategies facilitates interpretation of results as well as facilitating reproducibility and scalability [11]. The field of implementation science offers guidance on how to name and report these strategies [11]. Even though this study was unable to identify a single or gold standard approach to improving statin therapy for hypercholesterolemia disorders, it provides examples of many different approaches that have some impact on outcomes relevant to care. In this way, this study provides a roadmap for future implementation to better define implementation strategies and to rigorously define and test the outcomes associated with those strategies. More guidance will be needed on the impact of strategies in different healthcare settings, because different strategies may work better in different healthcare settings so these idiosyncrasies need to be understood.

\section{Conclusion}

Implementation strategies to improve the uptake of statins among patients with hypercholesterolemia exist but they are poorly reported and generalizability is limited. While these strategies lowered LDL-C and improved adherence, significant heterogeneity made assessment of the comparative effectiveness of strategies difficult. Future studies for increasing the utilization of statins among patients with hypercholesterolemia should more clearly define strategies used, prospectively test comparative effectiveness of different strategies, and use standardized efficacy endpoints.

\section{Abbreviations}

Cls: Confidence intervals; ERIC: Expert Recommendations for Implementing Change; LDL-C: Low density lipoprotein cholesterol; ORs: Odds ratios; PRIS MA: Preferred Reporting Items for Systematic Reviews and Meta-analyses; SMDs: Standardized mean differences; RCTs: Randomized clinical trails

\section{Supplementary Information}

The online version contains supplementary material available at https://doi. org/10.1186/s13012-021-01108-0.

Additional file 1: Appendix 1. Statin uptake search strategy. Appendix 2. Excluded full text articles and rationale. Appendix 3. Detailed study demographics. Appendix 4. Count of implementation strategy organized by category and strategy. Appendix 5. Detailed Proctor's framework description of each strategy. Appendix 6. Risk of bias

\section{Acknowledgements}

Not applicable.

\section{Authors' contributions}

LKJ designed the systematic review, reviewed abstracts and full-text, extracted and analyzed data, and prepared the manuscript file. ST and CG reviewed abstracts and full-text for inclusion and extracted data and reviewed manuscript file. LHY completed search and reviewed final manuscript file. YH performed statistical analyses and risk of bias and reviewed final manuscript. ACS, AKR, AG, RCB, and SSG reviewed data and reviewed final manuscript. TLS and TJW reviewed implementation strategies categorization and final manuscript. MSW and MRG designed systematic review, reviewed data, and prepared and reviewed final manuscript. All authors read and approved the final manuscript.

\section{Funding}

Research reported in this publication was supported by the National Heart, Lung, and Blood Institute of the National Institutes of Health under Award Number K12HL137942.

\section{Availability of data and materials}

All data generated or analyzed during this study are included in this published article and its supplementary information files.

\section{Declarations}

Ethics approval and consent to participate Not applicable. Registered in PROSPERO. 


\section{Consent for publication}

Not applicable.

\section{Competing interests}

The authors declare that they have no competing interests.

\section{Author details}

'Genomic Medicine Institute, Geisinger, 100 N Academy Ave., Danville, PA 17822, USA. ${ }^{2}$ Center for Pharmacy Innovation and Outcomes, Geisinger, Danville, PA, USA. ${ }^{3}$ Bernard Becker Medical Library, Washington University in St. Louis, St. Louis, MO, USA. ${ }^{4}$ Population Health Sciences, Geisinger, Danville, PA, USA. ${ }^{5}$ University of Health Sciences and Pharmacy in St. Louis, St. Louis, MO, USA. ${ }^{6}$ Population Health, Mercy Clinic_East Communities, St. Louis, MO, USA. ${ }^{7}$ Eastern Michigan University, Ypsilanti, MI, USA. ${ }^{8}$ Washington University School of Medicine, Washington University in St. Louis, St. Louis, MO, USA. ${ }^{9}$ Prevention Research Center in St. Louis, Brown School, Washington University in St. Louis, St. Louis, MO, USA. ${ }^{10}$ Department of Surgery (Division of Public Health Sciences) and Alvin J. Siteman Cancer Center, Washington University School of Medicine, Washington University in St. Louis, St. Louis, $\mathrm{MO}$, USA.

\section{Received: 29 September 2020 Accepted: 29 March 2021}

\section{Published online: 13 April 2021}

\section{References}

1. Grundy SM, Stone NJ, Bailey AL, et al. 2018 AHA/ACC/AACVPR/AAPA/ABC/ ACPM/ADA/AGS/APhA/ASPC/NLA/PCNA Guideline on the management of blood cholesterol: a report of the American College of Cardiology/American Heart Association Task Force on Clinical Practice Guidelines. J Am Coll Cardiol. 2019;139(25):e1082-143.

2. Akhabue E, Rittner SS, Carroll JE, et al. Implications of American College of Cardiology/American Heart Association (ACC/AHA) cholesterol guidelines on statin underutilization for prevention of cardiovascular disease in diabetes mellitus among several US networks of community health centers. J Am Heart Assoc. 2017;6(7):e005627.

3. Bansilal S, Castellano JM, Garrido E, Wei HG, Freeman A, Spettell C, et al. Assessing the impact of medication adherence on long-term cardiovascular outcomes. J Am Coll Cardiol. 2016;68(8):789-801. https://doi.org/10.1016/j.ja cc.2016.06.005.

4. Martin-Ruiz E, Olry-de-Labry-Lima A, Ocaña-Riola R, Epstein D. Systematic review of the effect of adherence to statin treatment on critical cardiovascular events and mortality in primary prevention. J Cardiovasc Pharmacol Ther. 2018;23(3):200-15. https://doi.org/10.1177/10742484177453 57.

5. De Vera MA, Bhole V, Burns LC, Lacaille D. Impact of statin adherence on cardiovascular disease and mortality outcomes: a systematic review. $\mathrm{Br}$ J Clin Pharmacol. 2014;78(4):684-98. https://doi.org/10.1111/bcp.12339.

6. Rodriguez F, Maron DJ, Knowles JW, Virani SS, Lin S, Heidenreich PA. Association of statin adherence with mortality in patients with atherosclerotic cardiovascular disease. JAMA Cardiol. 2019;4(3):206-13. https://doi.org/10.1001/jamacardio.2018.4936.

7. Jones LK, Sturm AC, Seaton TL, Gregor C, Gidding SS, Williams MS, et al. Barriers, facilitators, and solutions to familial hypercholesterolemia treatment. PLoS One. 2020;15(12):e0244193. https://doi.org/10.1371/journal. pone.0244193.

8. Tanner RM, Safford MM, Monda KL, Taylor B, O'Beirne R, Morris M, et al. Primary care physician perspectives on barriers to statin treatment. Cardiovasc Drugs Ther. 2017;31(3):303-9. https://doi.org/10.1007/s10557-01 7-6738-x.

9. Ju A, Hanson CS, Banks E, Korda R, Craig JC, Usherwood T, et al. Patient beliefs and attitudes to taking statins: systematic review of qualitative studies. Br J Gen Pract. 2018;68(671):e408-19. https://doi.org/10.3399/bjgp1 $8 \times 696365$.

10. Lansberg P, Lee A, Lee Z-V, Subramaniam K, Setia S. Nonadherence to statins: individualized intervention strategies outside the pill box. Vasc Health Risk Manag. 2018;14:91-102. https://doi.org/10.2147NHRM.S158641.

11. Proctor EK, Powell BJ, McMillen JC. Implementation strategies: recommendations for specifying and reporting. Implement Sci. 2013;8(1): 139. https://doi.org/10.1186/1748-5908-8-139.

12. Zamora A, Fernández De Bobadilla F, Carrion C, et al. Pilot study to validate a computer-based clinical decision support system for dyslipidemia treatment (HTE-DLP). Atherosclerosis. 2013;231(2):401-4. https://doi.org/10.1 016/j.atherosclerosis.2013.09.029.

13. Asch DA, Troxel AB, Stewart WF, Sequist TD, Jones JB, Hirsch AMG, et al. Effect of financial incentives to physicians, patients, or both on lipid levels: a randomized clinical trial. JAMA. 2015;314(18):1926-35. https://doi.org/10.1 001/jama.2015.14850.

14. Powell BJ, Waltz TJ, Chinman MJ, Damschroder LJ, Smith JL, Matthieu MM et al. A refined compilation of implementation strategies: results from the Expert Recommendations for Implementing Change (ERIC) project. Implement Sci. 2015;10(1):21. https://doi.org/10.1186/s13012-015-0209-1.

15. Moher D, Liberati A, Tetzlaff J, Altman DG. Preferred reporting items for systematic reviews and meta-analyses: the PRISMA statement. PLoS Med. 2009;6(7):e1000097. https://doi.org/10.1371/journal.pmed.1000097.

16. Waltz TJ, Powell BJ, Matthieu MM, Damschroder LJ, Chinman MJ, Smith JL, et al. Use of concept mapping to characterize relationships among implementation strategies and assess their feasibility and importance: results from the Expert Recommendations for Implementing Change (ERIC) study. Implement Sci. 2015;10(1):109. https:/doi.org/10.1186/s13012-015-0295-0.

17. Effective Practice and Organisation of Care (EPOC). 2015; epoc.cochrane.org/ epoc-taxonomy Accessed 1 Feb 2021.

18. Nau DP. Proportion of days covered (PDC) as a preferred method of measuring medication adherence: Pharmacy Quality Alliance; 2012.

19. Sterne JAC, Savovic J, Page MJ, et al. RoB 2: a revised tool for assessing risk of bias in randomised trials. BMJ. 2019;366:14898.

20. Sterne JA, Egger M. Funnel plots for detecting bias in meta-analysis: guidelines on choice of axis. J Clin Epidemiol. 2001;54(10):1046-55. https:// doi.org/10.1016/S0895-4356(01)00377-8.

21. Higgins JP, Thompson SG, Deeks JJ, Altman DG. Measuring inconsistency in meta-analyses. BMJ. 2003;327(7414):557-60. https://doi.org/10.1136/bmj.327. 7414.557.

22. Weng S, Kai J, Tranter J, Leonardi-Bee J, Qureshi N. Improving identification and management of familial hypercholesterolaemia in primary care: Preand post-intervention study. Atherosclerosis. 2018;274:54-60. https://doi. org/10.1016/j.atherosclerosis.2018.04.037.

23. Webster R, Li SC, Sullivan DR, Jayne K, Su SY, Neal B. Effects of internetbased tailored advice on the use of cholesterol-lowering interventions: a randomized controlled trial. J Med Internet Res. 2010;12(3):e42. https://doi. org/10.2196/jmir.1364.

24. Vrijens B, Belmans A, Matthys $K$, de Klerk E, Lesaffre E. Effect of intervention through a pharmaceutical care program on patient adherence with prescribed once-daily atorvastatin. Pharmacoepidemiol Drug Saf. 2006;15(2): 115-21. https://doi.org/10.1002/pds.1198.

25. Viola RA, Abbott KC, Welch PG, McMillan RJ, Sheikh AM, Yuan CM. A multidisciplinary program for achieving lipid goals in chronic hemodialysis patients. BMC Nephrol. 2002;3:1-7.

26. Vinker $\mathrm{S}$, Bitterman $\mathrm{H}$, Comaneshter D, Cohen AD. Physicians' behavior following changes in LDL cholesterol target goals. Isr J Health Policy Res. 2015;4(1):20.

27. Truppo C, Keller VA, Reusch MT, Pandya B, Bendich D. The effect of a comprehensive, mail-based motivational program for patients receiving lipid-lowering therapy. Manag Care Interface. 2003;16(3):35-40+62.

28. Straka RJ, Taheri R, Cooper SL, Smith JC. Achieving cholesterol target in a managed care organization (ACTION) trial. Pharmacotherapy. 2005;25(3): 360-71. https://doi.org/10.1592/phco.25.3.360.61601.

29. Stockl KM, Tjioe D, Gong S, Stroup J, Harada ASM, Lew HC. Effect of an intervention to increase statin use in medicare members who qualified for a medication therapy management program. J Manag Care Pharm. 2008; 14(6):532-40. https://doi.org/10.18553/jmcp.2008.14.6.532.

30. Stephenson SH, Larrinaga-Shum S, Hopkins PN. Benefits of the MEDPED treatment support program for patients with familial hypercholesterolemia. J Clin Lipidol. 2009;3(2):94-100. https://doi.org/10.1016/j.jacl.2009.02.004.

31. Stacy JN, Schwartz SM, Ershoff D, Shreve MS. Incorporating tailored interactive patient solutions using interactive voice response technology to improve statin adherence: results of a randomized clinical trial in a managed care setting. Popul Health Manage. 2009;12(5):241-54. https://doi. org/10.1089/pop.2008.0046.

32. Shibley MCH, Pugh CB. Implementation of pharmaceutical care services for patients with hyperlipidemias by independent community pharmacy practitioners. Ann Pharmacother. 1997;31(6):713-9. https://doi.org/10.1177/1 06002809703100608

33. Schwed A, Fallab CL, Burnier M, Waeber B, Kappenberger L, Burnand B, et al Electronic monitoring of compliance to lipid-lowering therapy in clinical 
practice. J Clin Pharmacol. 1999;39(4):402-9. https://doi.org/10.1177/00912 709922007976.

34. Schectman G, Wolff N, Byrd JC, Hiatt JG, Hartz A. Physician extenders for cost-effective management of hypercholesterolemia. J Gen Intern Med. 1996;11(5):277-86. https://doi.org/10.1007/BF02598268.

35. Riesen WF, Noll G, Dariolu R. Impact of enhanced compliance initiatives on the efficacy of rosuvastatin in reducing low density lipoprotein cholesterol levels in patients with primary hypercholesterolaemia. Swiss Med Wkly. 2008;138(29-30):420-6 DOI: 2008/29/smw-12120.

36. Rachmani R, Slavacheski I, Berla M, Frommer-Shapira R, Ravid M. Treatment of high-risk patients with diabetes: motivation and teaching intervention: a randomized, prospective 8-year follow-up study. J Am Soc Nephrol. 2005; 16(3 SUPPL. 1):S22-6.

37. Nordmann A, Blattmann L, Gallino A, Khetari R, Martina B, Müller P, et al. Systematic, immediate in-hospital initiation of lipid-lowering drugs during acute coronary events improves lipid control. Eur J Internal Med. 2000;11(6): 309-16. https://doi.org/10.1016/S0953-6205(00)00110-2.

38. Nguyen G, Cruickshank J, Mouillard A, Dumuis ML, Picard C, Cailleteau X, et al. Comparison of achievement of treatment targets as perceived by physicians and as calculated after implementation of clinical guidelines for the management of hypercholesterolemia in a randomized, clinical trial. Curr Ther Res Clin Exp. 2000;61(9):597-608. https://doi.org/10.1016/S0011-3 93X(00)88012-1.

39. Lindholm LH, Ekbom T, Dash C. Changes in cardiovascular risk factors by combined pharmacological and nonpharmacological strategies: the main results of the CELL study. J Intern Med. 1996;240(1):13-22. https://doi.org/1 0.1046/j.1365-2796.1996.492831000.x.

40. Lima EMO, Gualandro DM, Yu PC, Giuliano ICB, Marques AC, Calderaro D, et al. Cardiovascular prevention in HIV patients: results from a successful intervention program. Atherosclerosis. 2009;204(1):229-32. https://doi.org/1 0.1016/j.atherosclerosis.2008.08.017.

41. Lester WT, Grant RW, Barnett GO, Chueh HC. Randomized controlled trial of an informatics-based intervention to increase statin prescription for secondary prevention of coronary disease. J Gen Intern Med. 2006;21(1):229. https://doi.org/10.1111/j.1525-1497.2005.00268.x.

42. Kooy MJ, Van Wijk BLG, Heerdink ER, De Boer A, Bouvy ML. Does the use of an electronic reminder device with or without counseling improve adherence to lipid-lowering treatment? The results of a randomized controlled trial. Front Pharmacol. 2013:4:69.

43. Kardas P. An education-behavioural intervention improves adherence to statins. Cent Eur J Med. 2013;8(5):580-5.

44. Jakobsson S, Huber D, Björklund F, Mooe T. Implementation of a new guideline in cardiovascular secondary preventive care: subanalysis of a randomized controlled trial. BMC Cardiovasc Disord. 2016;16(1):1-9.

45. Hilleman DE, Faulkner MA, Monaghan MS. Cost of a pharmacist-directed intervention to increase treatment of hypercholesterolemia. Pharmacotherapy. 2004;24(8):1077-83. https://doi.org/10.1592/phco.24.11.1 077.36145.

46. Harrison TN, Green KR, Liu ILA, Vansomphone SS, Handler J, Scott RD, et al. Automated outreach for cardiovascular-related medication refill reminders. J Clin Hypertens. 2016;18(7):641-6. https://doi.org/10.1111/jch.12723.

47. Harats D, Leibovitz E, Maislos M, Wolfovitz E, Chajek-Shaul T, Leitersdorf E, et al. Cardiovascular risk assessment and treatment to target low density lipoprotein levels in hospitalized ischemic heart disease patients: results of the HOLEM study. Isr Med Assoc J. 2005;7(6):355-9.

48. Goldberg KC, Melnyk SD, Simel DL. Overcoming inertia: improvement in achieving target low-density lipoprotein cholesterol. Am J Manag Care. 2007;13(9):530-4.

49. Gitt AK, Jünger C, Jannowitz C, Karmann B, Senges J, Bestehorn K. Adherence of hospital-based cardiologists to lipid guidelines in patients at high risk for cardiovascular events (2L registry). Clin Res Cardiol. 2011;100(4): 277-87. https://doi.org/10.1007/s00392-010-0240-9.

50. Geber J, Parra D, Beckey NP, Korman L. Optimizing drug therapy in patients with cardiovascular disease: the impact of pharmacist-managed pharmacotherapy clinics in a primary care setting. Pharmacotherapy. 2002; 22(6 I):738-47.

51. Gavish D, Leibovitz E, Elly I, Shargorodsky M, Zimlichman R. Follow-up in a lipid clinic improves the management of risk factors in cardiovascular disease patients. Isr Med Assoc J. 2002;4(9):694-7.

52. Ford DR, Walker J, Game FL, Bartlett WA, Jones AF. Effect of computerized coronary heart disease risk assessment on the use of lipid-lowering therapy in general practice patients. Coron Health Care. 2001;5(1):4-8. https://doi. org/10.1054/chec.2000.0103.

53. Etxeberria A, Alcorta I, Pérez I, Emparanza Jl, Ruiz de Velasco E, Iglesias MT, et al. Results from the CLUES study: a cluster randomized trial for the evaluation of cardiovascular guideline implementation in primary care in Spain. BMC Health Serv Res. 2018;18(1):93. https://doi.org/10.1186/s12913-01 8-2863-X.

54. Mols RE, Jensen JM, Sand NP, et al. Visualization of coronary artery calcification: influence on risk modification. Am J Med. 2015;128(9):1023. e1023-31.

55. Dresser GK, Nelson SAE, Mahon JL, Zou G, Vandervoort MK, Wong CJ, et al. Simplified therapeutic intervention to control hypertension and hypercholesterolemia: a cluster randomized controlled trial (STITCH2). J Hypertens. 2013;31(8):1702-13. https://doi.org/10.1097/HJH.0b013e328361 9d6a.

56. de Velasco JA, Cosín J, de Oya M, de Teresa E. Intervention program to improve secondary prevention of Myocardial infarction. Results of the PRES ENTE (Early Secondary Prevention) study. Rev Esp Cardiol. 2004;57(2):146-54.

57. De Lusignan S, Belsey J, Hague N, Dhoul N, Van Vlymen J. Audit-based education to reduce suboptimal management of cholesterol in primary care: a before and after study. J Public Health. 2006;28(4):361-9. https://doi. org/10.1093/pubmed/fdl052.

58. Chung JST, Lee KKC, Tomlinson B, Lee WW. Clinical and economic impact of clinical pharmacy service on hyperlipidemic management in Hong Kong. J Cardiovasc Pharmacol Ther. 2011;16(1):43-52. https://doi.org/10.1177/1 074248410380207

59. Casebeer L, Huber C, Bennett N, et al. Improving the physician-patient cardiovascular risk dialogue to improve statin adherence. BMC Fam Pract. 2009;10:48. https://doi.org/10.1186/1471-2296-10-48.

60. Brath H, Morak J, Kästenbauer T, Modre-Osprian R, Strohner-Kästenbauer H, Schwarz M, et al. Mobile health (mHealth) based medication adherence measurement-a pilot trial using electronic blisters in diabetes patients. $\mathrm{Br}$ J Clin Pharmacol. 2013;76(S1):47-55. https://doi.org/10.1111/bcp.12184.

61. Bosworth HB, Brown JN, Danus S, Sanders LL, McCant F, Zullig LL, et al. Evaluation of a packaging approach to improve cholesterol medication adherence. Am J Manag Care. 2017;23(9):e280-6.

62. Bhattacharyya O, Harris S, Zwarenstein M, Barnsley J. Controlled trial of an intervention to improve cholesterol management in diabetes patients in remote Aboriginal communities. Int J Circumpolar Health. 2010;69(4):33343. https://doi.org/10.3402/ijch.v69i4.17629.

63. Bassa A, Del Val M, Cobos A, et al. Impact of a clinical decision support system on the management of patients with hypercholesterolemia in the primary healthcare setting. Dis Manag Health Outcomes. 2005;13(1):65-72. https://doi.org/10.2165/00115677-200513010-00007.

64. Andrews SB, Marcy TR, Osborn B, Planas LG. The impact of an appointmentbased medication synchronization programme on chronic medication adherence in an adult community pharmacy population. J Clin Pharm Ther. 2017;42(4):461-6. https://doi.org/10.1111/jcpt.12533.

65. Bogden PE, Koontz LM, Williamson P, Abbott RD. The physician and pharmacist team: An effective approach to cholesterol reduction. J Gen Intern Med. 1997;12(3):158-64.

66. Rehring TF, Stolcpart RS, Sandhoff BG, Merenich JA, Hollis HW Jr. Effect of a clinical pharmacy service on lipid control in patients with peripheral arterial disease. J Vasc Surg. 2006;43(6):1205-10. https://doi.org/10.1016/j.jvs.2006.02.019.

67. Hatfield J, Gulati S, Abdul Rahman MNA, Coughlin PA, Chetter IC. Nurse-led risk assessment/management clinics reduce predicted cardiac morbidity and mortality in claudicants. J Vasc Nurs. 2008;26(4):118-22. https://doi.org/1 0.1016/j.jvn.2008.09.004.

68. Aziz EF, Javed F, Pulimi S, Pratap B, de Benedetti Zunino ME, Tormey D, et al. Implementing a pathway for the management of acute coronary syndrome leads to improved compliance with guidelines and a decrease in angina symptoms. J Healthc Qual. 2012;34(4):5-14. https://doi.org/10.1111/ j.1945-1474.2011.00145.x.

69. Lappé JM, Muhlestein JB, Lappé DL, et al. Improvements in 1-year cardiovascular clinical outcomes associated with a hospital-based discharge medication program. Ann Intern Med. 2004;141(6):446-453+1-443.

70. Hilleman D, Monaghan M, Ashby C, Mashni J, Wooley K, Amato C. Simple physician-prompting intervention drastically improves outcomes in CHD. Formulary. 2002;37:209-10.

71. Faulkner MA, Wadibia EC, Lucas BD, Hilleman DE. Impact of pharmacy counseling on compliance and effectiveness of combination lipid-lowering 
therapy in patients undergoing coronary artery revascularization: a randomized, controlled trial. Pharmacotherapy. 2000;20(4):410-6. https://doi. org/10.1592/phco.20.5.410.35048.

72. Goswami NJ, DeKoven M, Kuznik A, et al. Impact of an integrated intervention program on atorvastatin adherence: a randomized controlled trial. Int J Gen Med. 2013;6:647-55. https://doi.org/10.2147/IJGM.S47518.

73. Clark B, DuChane J, Hou J, Rubinstein E, McMurray J, Duncan I. Evaluation of increased adherence and cost savings of an employer value-based benefits program targeting generic antihyperlipidemic and antidiabetic medications. J Manag Care Pharm. 2014;20(2):141-50. https://doi.org/10.18553/jmcp.2 014.20.2.141.

74. Farley JF, Wansink D, Lindquist JH, Parker JC, Maciejewski ML. Medication adherence changes following value-based insurance design. Am J Manag Care. 2012;18(5):265-74.

75. Chen C, Chen K, Hsu CY, Chiu WT, Li YC. A guideline-based decision support for pharmacological treatment can improve the quality of hyperlipidemia management. Comput Methods Prog Biomed. 2010;97(3): 280-5. https://doi.org/10.1016/j.cmpb.2009.12.004.

76. Choudhry NK, Isaac T, Lauffenburger JC, et al. Effect of a remotely delivered tailored multicomponent approach to enhance medication taking for patients with hyperlipidemia, hypertension, and diabetes the STIC2IT cluster randomized clinical trial. JAMA Intern Med. 2018;178(9):1190-8.

77. Khanal S, Obeidat O, Hudson MP, et al. Active lipid management in coronary artery disease (ALMICAD) study. Am J Med. 2007;120(8):734 e711-7.

78. Lee JK, Grace KA, Taylor AJ. Effect of a pharmacy care program on medication adherence and persistence, blood pressure, and low-density lipoprotein cholesterol: a randomized controlled trial. J Am Med Assoc. 2006;296(21):2563-71. https://doi.org/10.1001/jama.296.21.joc60162.

79. Damush TM, Myers L, Anderson JA, Yu Z, Ofner S, Nicholas G, et al. The effect of a locally adapted, secondary stroke risk factor selfmanagement program on medication adherence among veterans with stroke/TIA.[Erratum appears in Transl Behav Med. 2016 Sep;6(3):469; PMID: 27528534]. Trans| Behav Med. 2016;6(3):457-68. https://doi.org/1 0.1007/s13142-015-0348-6.

80. Mehrpooya M, Larki-Harchegani A, Ahmadimoghaddam D, et al. Evaluation of the effect of education provided by pharmacists on hyperlipidemic patient's adherence to medications and blood level of lipids. J Appl Pharm Sci. 2018;8(1):029-33.

81. Párraga-Martínez I, Escobar-Rabadán F, Rabanales-Sotos J, Lago-Deibe F, Téllez-Lapeira JM, Villena-Ferrer A, et al. Efficacy of a combined strategy to improve low-density lipoprotein cholesterol control among patients with hypercholesterolemia: a randomized clinical trial. Rev Esp Cardiol. 2018;71(1): 33-41. https://doi.org/10.1016/j.recesp.2017.03.019.

82. Patel A, Cass A, Peiris D, Usherwood T, Brown A, Jan S, et al. A pragmatic randomized trial of a polypill-based strategy to improve use of indicated preventive treatments in people at high cardiovascular disease risk. Eur J Prev Cardiol. 2015;22(7):920-30. https://doi.org/10.1177/2047487314530382.

83. McAlister FA, Majumdar SR, Padwal RS, et al. Case management for blood pressure and lipid level control after minor stroke: PREVENTION randomized controlled trial. CMAJ. 2014;186(8):577-84. https://doi.org/10.1503/cmaj.140053.

84. Shoulders BR, Franks AS, Barlow PB, Williams JD, Farland MZ. Impact of pharmacists' interventions and simvastatin dose restrictions. Ann Pharmacother. 2014;48(1):54-61. https://doi.org/10.1177/1060028013511323.

85. Derose SF, Green K, Marrett E, Tunceli K, Cheetham TC, Chiu VY, et al. Automated outreach to increase primary adherence to cholesterol-lowering medications. JAMA Intern Med. 2013;173(1):38-43. https://doi.org/10.1001/2 013.jamainternmed.717.

86. Nieuwkerk PT, Nierman MC, Vissers MN, Locadia M, Greggers-Peusch P, Knape LPM, et al. Intervention to improve adherence to lipid-lowering medication and lipid-levels in patients with an increased cardiovascular risk. Am J Cardiol. 2012;110(5):666-72. https://doi.org/10.1016/j.amjcard.2 012.04.045.

87. Schmittdiel JA, Karter AJ, Dyer W, Parker M, Uratsu C, Chan J, et al. The comparative effectiveness of mail order pharmacy use vs. local pharmacy use on LDL-C control in new statin users. J Gen Intern Med. 2011;26(12): 1396-402. https://doi.org/10.1007/s11606-011-1805-7.

88. Coodley GO, Jorgensen M, Kirschenbaum J, Sparks C, Zeigler L, Albertson BD. Lowering LDL Cholesterol in adults: a prospective, community-based practice initiative. Am J Med. 2008;121(7):604-10. https://doi.org/10.1016/j.a mjmed.2008.02.031.
89. Willich SN, Englert H, Sonntag F, Völler H, Meyer-Sabellek W, Wegscheider $\mathrm{K}$, et al. Impact of a compliance program on cholesterol control: Results of the randomized ORBITAL study in 8108 patients treated with rosuvastatin. Eur J Prev Cardiol. 2009;16(2):180-7. https:// doi.org/10.1097/HJR.0b013e3283262ac3.

90. Hung CS, Lin JW, Hwang JJ, Tsai RY, Li AT. Using paper chart based clinical reminders to improve guideline adherence to lipid management. J Eval Clin Pract. 2008;14(5):861-6. https://doi.org/10.1111/j.1365-2753.2008.01066.x.

91. Brady AJB, Pittard JB, Grace JF, Robinson PJ. Clinical assessment alone will not benefit patients with coronary heart disease: failure to achieve cholesterol targets in 12,045 patients - The Healthwise II study. Int J Clin Pract. 2005;59(3):342-5. https://doi.org/10.1111/j.1742-1241.2005.00365.x.

92. Robinson JG, Conroy C, Wickemeyer WJ. A novel telephone-based system for management of secondary prevention to a low-density lipoprotein cholesterol < or = 100 mg/dl. Am J Cardiol. 2000;85(3):305-8. https://doi. org/10.1016/S0002-9149(99)00737-7.

93. Birtcher KK, Bowden C, Ballantyne CM, Huyen M. Strategies for implementing lipid-lowering therapy: pharmacy-based approach. Am J Cardiol. 2000;85(3 SUPPL. 1):30-5.

94. Shaffer J, Wexler LF. Reducing low-density lipoprotein cholesterol levels in an ambulatory care system: results of a multidisciplinary collaborative practice lipid clinic compared with traditional physician-based care. Arch Intern Med. 1995;155(21):2330-5. https://doi.org/10.1001/archinte.1995.0043 0210080012.

95. Choe HM, Stevenson JG, Streetman DS, Heisler M, Sandiford CJ, Piette JD. Impact of patient financial incentives on participation and outcomes in a statin pill-splitting program. Am J Manag Care. 2007;13(6 Part 1):298-304.

96. McLeod AL, Brooks L, Taylor V, Wylie A, Currie PF, Dewhurst NG. Nonattendance at secondary prevention clinics: the effect on lipid management. Scott Med J. 2005;50(2):54-6. https://doi.org/10.1177/0036933 00505000204

97. Rabinowitz I, Tamir A. The SaM (Screening and Monitoring) approach to cardiovascular risk-reduction in primary care-cyclic monitoring and individual treatment of patients at cardiovascular risk using the electronic medical record. Eur J Cardiovasc Prev Rehabil. 2005;12(1):56-62.

98. Ryan MJ, Gibson J, Simmons P, Stanek E. Effectiveness of aggressive management of dyslipidemia in a collaborative-care practice model. Am J Cardiol. 2003;91(12):1427-31. https://doi.org/10.1016/S0002-9149(03)00393-X.

99. Sebregts EH, Falger PR, Bär FW, Kester AD, Appels A. Cholesterol changes in coronary patients after a short behavior modification program. Int J Behav Med. 2003;10(4):315-30. https://doi.org/10.1207/S15327558IJBM1004_3.

100. Lowrie R, Lloyd SM, McConnachie A, Morrison J. A cluster randomised controlled trial of a pharmacist-led collaborative intervention to improve statin prescribing and attainment of cholesterol targets in primary care. PLoS One. 2014;9(11):e113370. https://doi.org/10.1371/journal.pone.0113370.

101. McAlister FA, Fradette M, Majumdar SR, et al. The enhancing secondary prevention in coronary artery disease trial. CMAJ. 2009;181(12):897-904. https://doi.org/10.1503/cmaj.090917.

102. Persell SD, Shah S, Brown T, et al. Individualized risk communication and lay outreach for the primary prevention of cardiovascular disease in community health centers: a randomized controlled trial. Circulation. 2015;130:A14008.

103. Villeneuve J, Genest J, Blais L, Vanier MC, Lamarre D, Fredette M, et al. A cluster randomized controlled trial to evaluate an ambulatory primary care management program for patients with dyslipidemia: the TEAM study. CMAJ. 2010;182(5):447-55. https://doi.org/10.1503/cmaj.090533.

104. Osborn D, Burton A, Hunter R, Marston L, Atkins L, Barnes T, et al. Clinical and cost-effectiveness of an intervention for reducing cholesterol and cardiovascular risk for people with severe mental illness in English primary care: a cluster randomised controlled trial. Lancet Psychiatry. 2018;5(2):14554. https://doi.org/10.1016/\$2215-0366(18)30007-5.

105. Paulós CP, Akesson Nygren CE, Celedón C, Cárcamo CA. Impact of a pharmaceutical care program in a community pharmacy on patients with dyslipidemia. Ann Pharmacother. 2005;39(5):939-43. https://doi.org/10.134 5/aph.1E347.

106. Briss PA, Brownson RC, Fielding JE, Zaza S. Developing and using the guide to community preventive services: lessons learned about evidence-based public health. Annu Rev Public Health. 2004;25(1):281-302. https://doi.org/1 0.1146/annurev.publhealth.25.050503.153933.

107. Powell BJ, Fernandez ME, Williams NJ, et al. Enhancing the Impact of Implementation Strategies in Healthcare: A Research Agenda. Front Public Health. 2019;7:3. https://doi.org/10.3389/fpubh.2019.00003. 
108. Powell BJ, McMillen JC, Proctor EK, et al. A compilation of strategies for implementing clinical innovations in health and mental health. Med Care Res Rev. 2012;69(2):123-57. https://doi.org/10.1177/1077558711430690.

109. Graham AK, Lattie EG, Powell BJ, Lyon AR, Smith JD, Schueller SM, et al. Implementation strategies for digital mental health interventions in health care settings. Am Psychol. 2020;75(8):1080-92. https://doi.org/10.1037/amp0000686.

110. Trogrlić $Z$, van der Jagt $M$, Bakker J, et al. A systematic review of implementation strategies for assessment, prevention, and management of ICU delirium and their effect on clinical outcomes. Crit Care. 2015;19(1):157.

111. Yi Mohammadi JJ, Franks $K$, Hines S. Effectiveness of professional oral health care intervention on the oral health of residents with dementia in residential aged care facilities: a systematic review protocol. JBI Database System Rev Implement Rep. 2015;13(10):110-22. https://doi.org/10.11124/jbisrir-2015-2330.

112. Mills KT, Obst KM, Shen W, Molina S, Zhang HJ, He H, et al. Comparative effectiveness of implementation strategies for blood pressure control in hypertensive patients: a systematic review and meta-analysis. Ann Intern Med. 2018;168(2):110-20. https://doi.org/10.7326/M17-1805.

113. Kinnear FJ, Wainwright E, Perry R, Lithander FE, Bayly G, Huntley A, et al. Enablers and barriers to treatment adherence in heterozygous familial hypercholesterolaemia: a qualitative evidence synthesis. BMJ Open. 2019; 9(7):e030290. https://doi.org/10.1136/bmjopen-2019-030290.

114. Ament SM, de Groot JJ, Maessen JM, Dirksen CD, van der Weijden T, Kleijnen J. Sustainability of professionals' adherence to clinical practice guidelines in medical care: a systematic review. BMJ Open. 2015;5(12): e008073. https://doi.org/10.1136/bmjopen-2015-008073.

115. Jordan P, Mpasa F, Ten Ham-Baloyi W, Bowers C. Implementation strategies for guidelines at ICUs: a systematic review. Int J Health Care Qual Assur. 2017;30(4):358-72. https://doi.org/10.1108/JHCQA-08-2016-0119.

116. Unverzagt S, Oemler M, Braun K, Klement A. Strategies for guideline implementation in primary care focusing on patients with cardiovascular disease: a systematic review. Fam Pract. 2014;31(3):247-66. https://doi.org/1 0.1093/fampra/cmt080

117. Shanbhag D, Graham ID, Harlos K, Haynes RB, Gabizon I, Connolly SJ, et al. Effectiveness of implementation interventions in improving physician adherence to guideline recommendations in heart failure: a systematic review. BMJ Open. 2018;8(3):e017765. https://doi.org/10.1136/bmjopen-2017-017765.

118. Milchak JL, Carter BL, James PA, Ardery G. Measuring adherence to practice guidelines for the management of hypertension: an evaluation of the literature. Hypertension. 2004;44(5):602-8.

119. Morrison A, Polisena J, Husereau D, Moulton K, Clark M, Fiander M, et al. The effect of English-language restriction on systematic review-based metaanalyses: a systematic review of empirical studies. Int J Technol Assess Health Care. 2012;28(2):138-44. https://doi.org/10.1017/S0266462312000086.

120. Schmucker CM, Blumle A, Schell LK, et al. Systematic review finds that study data not published in full text articles have unclear impact on metaanalyses results in medical research. PLoS One. 2017;12(4):e0176210. https:// doi.org/10.1371/journal.pone.0176210.

121. Bays HE, Jones PH, Brown WW, Jacobson TA, National LA. National lipid association annual summary of clinical lipidology 2015. J Clin Lipidol. 2014; 8(6 Suppl):S1-36. https://doi.org/10.1016/j.jacl.2014.10.002.

122. Williamson PR, Altman DG, Bagley H, et al. The COMET Handbook: version 1. 0. Trials. 2017;18(3):280.

123. Seidler AL, Hunter KE, Cheyne S, Ghersi D, Berlin JA, Askie L. A guide to prospective meta-analysis. BMJ. 2019;367:15342.

\section{Publisher's Note}

Springer Nature remains neutral with regard to jurisdictional claims in published maps and institutional affiliations.

Ready to submit your research? Choose BMC and benefit from:
- fast, convenient online submission
- thorough peer review by experienced researchers in your field
- rapid publication on acceptance
- support for research data, including large and complex data types
- gold Open Access which fosters wider collaboration and increased citations
- maximum visibility for your research: over 100M website views per year
At BMC, research is always in progress.
Learn more biomedcentral.com/submissions

